\title{
ALTERACIONES METABÓLICAS EN ESCOLARES CON OBESIDAD, CONSULTA DE ENDOCRINOLOGÍA PEDIÁTRICA, CLÍNICA DOCTOR VIRGILIO CEDANO. ENERO-JUNIO 2019
}

\author{
Metabolic Alterations in Schoolchildren with obesity, Pediatric \\ Endocrinology Deparment, Doctor Virgilio Cedano Clinic. \\ January-June 2019
}

\author{
Cedano Mariellaa, Díaz Ángel ${ }^{b},{\text { Almánzar Rosarioc }{ }^{c} \text { Mercedes Ramos }}^{d}$
}

Recibido: 6 de diciembre, 2019 • Aprobado: 14 de febrero, 2020

Cómo citar: Mariella C, Ángel D, Rosario A, Ramos M. Alteraciones metabólicas en escolares con obesidad: consulta de Endocrinología Pediátrica, Clínica doctor Virgilio Cedano. enero-junio 2019. cysa [Internet]. 16 de junio de 2020 [citado 17 de junio de 2020];4(2):65-70. Disponible en: https://revistas.intec.edu.do/index.php/cisa/article/view/1762

\section{Resumen}

El objetivo del estudio fue determinar las alteraciones metabólicas presentes en los escolares con obesidad que asisten a la consulta de Endocrinología Pediátrica de la Clínica Doctor Virgilio Cedano, desde enero hasta junio 2019, en el municipio de Higüey, provincia La Altagracia. Se realizó un estudio descriptivo, observacional, de corte transversal con colección retrospectiva de datos, donde fueron evaluados 68 expedientes clínicos durante el período enero-julio 2019. Al finalizar la investigación, se concluye que las principales alteraciones metabólicas encontradas en los escolares con obesidad fueron la acantosis nigricans, la deficiencia de vitamina $\mathrm{D}$ y las dislipidemias. El sexo femenino fue el más frecuente. El $38 \%$ de los pacientes obesos tuvieron antecedentes familiares patológicos de Diabetes Mellitus tipo 2. El 49 \% de la población estudiada tiene Insulinorresistencia. La mayoría de los escolares obesos tienen obesidad central. El $48 \%$ de los pacientes con obesidad central tiene insulinorresistencia. La resistencia a la insulina está presente en la mayoría de los pacientes con alteraciones metabólicas.

Palabras clave: alteración metabólica; escolares; obesidad; insulinorresistencia.

\footnotetext{
a Pediatra Endocrinóloga, Profesora Bioquímica, Universidad Autónoma de Santo Domingo, Santo Domingo, República Dominicana Correo-e: p991533@uasd.edu.do, mariella_cn@hotmail.com ORCID: 0000-00017446-7357

b Nutriólogo Clínico, Profesor Bioquímica, Universidad Autónoma de Santo Domingo, República Dominicana. Correo-e: asdb@hotmail.com
}

\begin{abstract}
The objective of the study was to determine the metabolic alterations present in schoolchildren with obesity who attend the Pediatric Endocrinology clinic of the Doctor Virgilio Cedano Clinic from January to June 2019, in the municipality of Higüey in the province of La Altagracia. A descriptive, observational, cross-sectional study with retrospective data collection was conducted, where 68 clinical records were evaluated during the period JanuaryJuly 2019. At the end of the investigation, it is concluded that the main metabolic alterations found in schoolchildren with obesity were acanthosis nigricans, vitamin $\mathrm{D}$ deficiency and dyslipidemias. The female sex was the most frequent. $38 \%$ of obese patients had a family history of type 2 diabetes. Insulinorresistencia has $49 \%$ of the population studied. Most obese schoolchildren have central obesity. $48 \%$ of patients with central obesity have insulin resistance. Insulin resistance is present in most patients with metabolic disorders.
\end{abstract}

Keywords: Metabolic alterations; school; obesity; insulin resistance.

\footnotetext{
${ }^{\mathrm{c}}$ Pediatra Endocrinóloga, Hospital Infantil Doctor Robert Reid Cabral, República Dominicana. Correo-e: rosarioalmanzar@gmail.com

d Residente Endocrinología Pediátrica, Hospital Infantil Doctor Robert Reid Cabral, República Dominicana.

Correo-e: ramos-mercedes@hotmail.com
} 


\section{Introducción}

La obesidad consiste en la acumulación excesiva de tejido adiposo. Su definición clínica se realiza por medio de la estimación del índice de masa corporal (IMC). Los niños y adolescentes obesos tienen mayor riesgo de padecer las complicaciones asociadas a la obesidad como alteraciones metabólicas a edades más tempranas.

De acuerdo con la evidencia científica se confirma el aumento alarmante de la obesidad en niños y adolescentes en los últimos años. Su etiología se basa en factores genéticos y ambientales, donde se destaca el aumento de la ingesta calórica y la disminución de la actividad física ${ }^{1,2,3,4}$. Dentro de las complicaciones metabólicas que predominan en la población escolar obesa sobresale el eje cardinal de la resistencia a la insulina asociada a la acantosis nigricans, intolerancia a los hidratos de carbono, diabetes mellitus tipo 2, dislipidemias, esteatosis hepática, síndrome de ovario poliquísticos y síndrome metabólico ${ }^{5}$.

Diversos estudios señalan la presencia de dislipidemia, resistencia a la insulina y deficiencia de vitamina $\mathrm{D}$ en niños con obesidad ${ }^{3,6,7}$, esto se traduce a mayor riesgo de desarrollar diabetes mellitus tipo 2 a una edad más temprana y aumenta el riesgo cardiovascular.

En la evaluación metabólica de los niños obesos, el perfil bioquímico debe incluir la medición de los niveles de insulina, en ayunas y 2 horas después de la sobrecarga de glucosa, si se encuentran elevados, se estaría frente a una hiperinsulinemia que aunado a los valores de la glicemia, permiten calcular el índice $\mathrm{HOMA}^{8}$, herramienta útil para confirmar la resistencia a la insulina. Una vez detectada la RI, la intervención terapéutica oportuna permitiría prevenir la aparición de complicaciones como la diabetes mellitus tipo 2, dislipidemia y aterosclerosis.
En la revisión bibliográfica nacional, no se encontraron antecedentes de investigaciones sobre las alteraciones metabólicas en niños y adolescentes obesos en la región este del país. Partiendo de la premisa planteada, se formula la siguiente interrogante: ¡cuáles son las alteraciones metabólicas de los escolares con obesidad que asisten a la consulta de Endocrinología Pediátrica de la Clínica Dr. Virgilio Cedano durante el período enero-junio 2019?

\section{Objetivo general}

Determinar las alteraciones metabólicas presentes en los escolares con obesidad que asisten a la consulta de Endocrinología Pediátrica de la Clínica Doctor Virgilio Cedano desde enero hasta junio 2019.

\section{Objetivos específicos}

- Identificar las alteraciones metabólicas según el sexo y la edad de los escolares obesos.

- Relacionar las alteraciones metabólicas con los antecedentes familiares patológicos en los escolares con obesidad.

- Determinar la presencia de insulinorresistencia en la población de estudio.

- Establecer la relación entre la obesidad central y la insulinorresistencia de los pacientes estudiados.

- Relacionar la resistencia a la insulina con las alteraciones metabólicas en escolares con obesidad.

\section{Material y métodos}

Se realizó un estudio descriptivo, observacional de corte transversal y colección retrospectiva de datos, con el objetivo de establecer las alteraciones 
metabólicas en escolares con obesidad entre 5 y 16 años que asisten a la consulta de Endocrinología Pediátrica de la Clínica Doctor Virgilio Cedano, de Higüey, durante el semestre enero-junio del 2019. El universo estuvo formado por los expedientes de pacientes de ambos sexos con obesidad exógena en el período de estudio. Se tomó una muestra no probabilística por conveniencia.

Criterios de inclusión:

- Expedientes de pacientes obesos (con índice de masa corporal igual o mayor al 95 percentil para edad y sexo)

- De ambos sexos entre 5 y 16 años

Criterios de exclusión:

- Pacientes con obesidad de causa no exógena

- Expedientes incompletos

Se revisó la base de datos de las historias clínicas de los pacientes diagnosticados desde enero hasta junio 2019. Esta revisión se realizó durante el mes de junio con autorización previa del personal médico de la consulta.

Para evaluación de los antecedentes familiares, patológicos, se consideraron las enfermedades de diabetes mellitus tipo 2 e hipertensión arterial en los familiares de primer grado de consaguinidad (madre y padre) y segundo grado (abuelos maternos y paternos). La insulinorresistencia se midió utilizando como parámetro el valor por encima de 3.1 del índice HOMA, que se calcula con la fórrmula de glicemia basal (mg/dl) por insulina basal entre 405 . La obesidad central fue estimada con la tabla de la circunferencia abdominal en niños y niñas del Estudio ENKID (de España), tomando en cuenta el perímetro abdominal mayor del 90 percentil para edad y sexo.
En cuanto a las alteraciones metabólicas, para establecer la deficiencia de vitamina $\mathrm{D}$, en los pacientes estudiados, se incluyeron aquellos con valores de la $25 \mathrm{OH}$ vitamina $\mathrm{D}$ total menores de $30 \mathrm{ng} / \mathrm{mL}$. La presencia de dislipidemia se estableció con los parámetros de triacilgliceroles mayores de $110 \mathrm{mg} /$ $\mathrm{dL}$ y/o colesterol total mayores de $170 \mathrm{mg} / \mathrm{dL}$. El diagnóstico de esteatosis hepática se confirmó por ultrasonografía de abdomen en los expedientes.

\section{Resultados}

Tabla 1. Alteraciones metabólicas de los escolares con obesidad. Consulta de Endocrinología Pediátrica, Clínica Doctor Virgilio Cedano, enero-junio 2019

\begin{tabular}{|c|c|c|}
\hline Alteraciones metabólicas & $\begin{array}{c}\text { Número de } \\
\text { casos }\end{array}$ & Porcentaje \\
\hline Acantosis Nigricans & 51 & $75 \%$ \\
\hline Deficiencia de Vitamina D & 29 & $43 \%$ \\
\hline Dislipidemias & 24 & $35 \%$ \\
\hline Esteatosis Hepática & 5 & $7 \%$ \\
\hline $\begin{array}{c}\text { Síndrome de Ovario } \\
\text { Poliquístico }\end{array}$ & 5 & $7 \%$ \\
\hline $\begin{array}{c}\text { Intolerancia a los Hidratos de } \\
\text { Carbono }\end{array}$ & 1 & $1 \%$ \\
\hline
\end{tabular}

Fuente: elaboración propia, a partir de los expedientes clínicos del archivo de la Consulta de Endocrinología Pediátrica. Clínica Dr. Virgilio Cedano.

Tabla 2. Antecedentes familiares patológicos de los escolares obesos. Consulta de Endocrinología Pediátrica, Clínica Doctor Virgilio Cedano, enero-junio 2019

\begin{tabular}{|c|c|c|}
\hline $\begin{array}{c}\text { Antecedentes familiares } \\
\text { patológicos }\end{array}$ & Frecuencia & Porcentaje \\
\hline Diabetes Mellitus tipo 2 & 26 & $38 \%$ \\
\hline Hipertensión Arterial & 38 & $56 \%$ \\
\hline
\end{tabular}

Fuente: elaboración propia a partir de los expedientes clínicos del archivo de la Consulta de Endocrinología Pediátrica. Clínica Dr. Virgilio Cedano. 
Tabla 3. Insulinorresistencia en escolares obesos. Consulta de Endocrinología Pediátrica, Clínica Doctor Virgilio Cedano, enero-junio 2019

\begin{tabular}{|c|c|c|}
\hline Índice HOMA & $\begin{array}{c}\text { Número de } \\
\text { casos }\end{array}$ & Porcentaje \\
\hline$\geq 3.1$ & 33 & $49 \%$ \\
\hline$<3.1$ & 35 & $51 \%$ \\
\hline Total & 68 & $100 \%$ \\
\hline
\end{tabular}

Fuente: elaboración propia a partir de los expedientes clínicos del archivo de la Consulta de Endocrinología Pediátrica. Clínica Dr. Virgilio Cedano.

Tabla 4. Obesidad central en escolares obesos. Consulta de Endocrinología Pediátrica, Clínica Doctor Virgilio Cedano, enero-junio 2019

\begin{tabular}{|c|c|c|}
\hline $\begin{array}{c}\text { Perímetro } \\
\text { Abdominal }\end{array}$ & Número de casos & Porcentaje \\
\hline$\geq 90 \mathrm{p}$ & 66 & $97 \%$ \\
\hline$<90 \mathrm{p}$ & 2 & $3 \%$ \\
\hline Total & 68 & $100 \%$ \\
\hline
\end{tabular}

Fuente: elaboración propia a partir de los expedientes clínicos del archivo de la Consulta de Endocrinología Pediátrica, Clínica Dr. Virgilio Cedano.

Tabla 5. Relación entre la obesidad central y la resistencia a la insulina en escolares obesos. Consulta de Endocrinología Pediátrica, Clínica Doctor Virgilio Cedano, enero-junio 2019

\begin{tabular}{|c|c|c|c|}
\hline $\begin{array}{c}\text { Perímetro } \\
\text { Abdominal }\end{array}$ & $\begin{array}{c}\text { Número } \\
\text { de casos }\end{array}$ & $\begin{array}{c}\text { Insulinorresis- } \\
\text { tencia }\end{array}$ & Porcentaje \\
\hline$\geq 90 \mathrm{p}$ & 66 & 32 & $48 \%$ \\
\hline$<90 \mathrm{p}$ & 2 & 1 & $50 \%$ \\
\hline Total & 68 & 33 & $49 \%$ \\
\hline
\end{tabular}

Fuente: elaboración propia a partir de los expedientes clínicos del archivo de la Consulta de Endocrinología Pediátrica. Clínica Dr. Virgilio Cedano.
Cuadro 6. Relación entre la resistencia a la insulina y las alteraciones metabólicas en escolares obesos. Consulta de Endocrinología Pediátrica, Clínica Doctor Virgilio Cedano, enero-junio 2019

\begin{tabular}{|c|c|c|c|}
\hline $\begin{array}{c}\text { Alteraciones } \\
\text { Metabólicas }\end{array}$ & $\begin{array}{c}\text { Número } \\
\text { de casos }\end{array}$ & $\begin{array}{c}\text { Insulinor- } \\
\text { resistencia }\end{array}$ & Porcentaje \\
\hline $\begin{array}{c}\text { Acantosis } \\
\text { Nigricans }\end{array}$ & 51 & 27 & $53 \%$ \\
\hline $\begin{array}{c}\text { Déficit de } \\
\text { Vitamina D }\end{array}$ & 29 & 14 & $48 \%$ \\
\hline Dislipidemias & 24 & 13 & $54 \%$ \\
\hline $\begin{array}{c}\text { Esteatosis } \\
\text { Hepática }\end{array}$ & 5 & 2 & $40 \%$ \\
\hline $\begin{array}{c}\text { Síndrome } \\
\text { de Ovario } \\
\text { Poliquístico }\end{array}$ & 5 & 4 & $80 \%$ \\
\hline $\begin{array}{c}\text { Intolerancia a } \\
\text { los Hidratos de } \\
\text { Carbono }\end{array}$ & 1 & 1 & $100 \%$ \\
\hline
\end{tabular}

Fuente: elaboración propia a partir de los expedientes clínicos del archivo de la Consulta de Endocrinología Pediátrica. Clínica Dr. Virgilio Cedano.

\section{Discusión}

Se revisaron 68 expedientes de pacientes en edad escolar diagnosticados con obesidad, en la consulta de Endocrinología Pediátrica de la Clínica Doctor Virgilio Cedano, desde enero hasta junio 2016.

Dentro de las alteraciones metabólicas (ver tabla 1), la acantosis nigricans estuvo presente en el $75 \%$ de los casos, coincidiendo con el estudio de Rivero ${ }^{7}$ et al., donde el $55 \%$ de los pacientes estudiados tenía acantosis nigricans al examen físico. La deficiencia de la vitamina $\mathrm{D}$ se encontró en el $43 \%$ de los escolares con obesidad, que se relaciona con los valores del estudio de Erol et al., ${ }^{6}$ donde el $69 \%$ de los pacientes presentó niveles de vitamina $\mathrm{D}$ por debajo $20 \mathrm{ng} / \mathrm{dL}$. Mientras que las dislipidemias estuvieron en el $35 \%$ de los escolares obesos, estos resultados también coinciden con el Erol et al., donde el 36,1 \% 
de su población de niños obesos tenían dislipidemias. La esteatosis hepática, el síndrome de ovario poliquístico y la intolerancia a los hidratos de carbono fueron menos frecuentes en la población estudiada. No se encontraron casos con Diabetes Mellitus tipo 2.

De los escolares obesos, el $71 \%$ de los pacientes correspondió al sexo femenino, de las cuales el $75 \%$ tenía acantosis nigricans, el $44 \%$ deficiencia de vitamina $\mathrm{D}$, el $40 \%$ dislipidemias, como alteraciones metabólicas más relevantes según el sexo. Mientras que el $29 \%$ de los pacientes fueron del sexo masculino, mostrando valores similares de acantosis nigricans $(75 \%)$ y deficiencia de vitamina D (40 \%) y menor frecuencia de dislipidemia $(25 \%)$.

Los pacientes se clasificaron en categorías según su edad que abarcó un rango desde 5 hasta los 16 años, con una media de 10 de $10.2+0.3$ años, para un intervalo de confianza de $95 \%$ y una desviación estándar de 2.5 años.

El 41\% de los casos revisados correspondió al grupo etario entre 8 a 10 años, de los cuales el $75 \%$ presentó acantosis nigricans al momento de la evaluación física inicial, el $57 \%$ tuvo deficiencia de vitamina D, y el $46 \%$ dislipidemias. Mientras que el $29 \%$ de los casos revisados se encontró entre 11 y 13 años, sin diferencias significativas en cuanto a las alteraciones metabólicas. Los antecedentes familiares patológicos de diabetes mellitus tipo 2, en el primer y segundo grado de consanguinidad se encontraron en el $38 \%$ de los escolares obesos.

La insulinorresistencia estuvo presente en el $49 \%$ de la población estudiada (ver tabla 3 ), a diferencia de los hallazgos de Martos et al., donde el $20 \%$ de los pacientes estudiados presentó resistencia a la insulina. En cuanto a la obesidad central (tabla 4), el $97 \%$ de los pacientes tuvo un perímetro abdominal mayor al 90 percentil para edad y sexo. De los pacientes con obesidad central, el $48 \%$ presentó insulinorresistencia (ver tabla 5).

Cuando se establece la relación entre la resistencia a la insulina con las alteraciones metabólicas (ver tabla 6), se observó que el $80 \%$ de las pacientes con síndrome de ovario poliquístico, el $54 \%$ de los pacientes con dislipidemias, el $53 \%$ de los que presentaron acantosis nigricans, el $48 \%$ de los que presentaron deficiencia de vitamina $\mathrm{D}$, y el $40 \%$ de los pacientes con esteatosis hepática presentaron un Índice HOMA mayor a 3.1, confirmando la presencia de insulinorresistencia.

\section{Conclusiones}

Las principales alteraciones metabólicas encontradas en los escolares con obesidad fueron la acantosis nigricans, la deficiencia de vitamina $\mathrm{D}$ y las dislipidemias.

El sexo femenino fue el más frecuente.

El $75 \%$ de las pacientes presentó acantosis nigricans y el $44 \%$ tenía deficiencia de vitamina D.

El grupo etario más frecuente fue el rango de 8 a 10 años.

El $38 \%$ de los pacientes obesos tuvieron antecedentes familiares patológicos de Diabetes Mellitus tipo 2.

El 49 \% de la población estudiada tiene insulinorresistencia.

La mayoría de los escolares obesos tienen obesidad central.

El $48 \%$ de los pacientes con obesidad central tiene insulinorresistencia.

La resistencia a la insulina está presente en la mayoría de los pacientes con alteraciones metabólicas. 


\section{Bibliografía}

1. Raimann X. Obesidad y sus complicaciones. Rev Med. Cli Los Andes. 2011;22(1):4-141. Available from: https://www.sciencedirect.com/ science/article/pii/S0716864011703893

2. Kumar S, Kelly A. Review of Childhood Obesity: From Epidemiology, Etiology and Comorbidities to Clinical Assessment and Treatment. Mayo Clin Proc. 2017;92(2):251-65.

3. Gunkor N. Sobrepeso y obesidad en niños y adolescentes. J Clin Res Pediatr Endocrinol. 2014;6(3):29-43. Available from https://www. ncbi.nlm.nih.gov/pubmed/25241606

4. Vikram N. Cardiovascular and metabolic complications-diagnosis and management in obese children. Indian J Pediatr. 2018;85(7):535-45. Available from https://www.ncbi.nlm.nih.gov/ pubmed/2921864

5. Erol M, Bostan G, Hamilçıkan Ş, Can E, Yiğit Ö. La deficiencia de vitamina $\mathrm{D}$ y la resistencia a la insulina como factores de riesgo de dislipidemia en niños obesos. Arch. argent. pediatr. 2017;115(2):133-9. Available: http://www. scielo.org.ar/scielo.php?script=sci_arttext\&pid= S0325-00752017000200006\&lng=es

6. Rivero G, Cabrera P, Álvarez M, Pantoja O. Alteraciones metabólicas en pacientes obesos y su asociación con la acantosis nigricans. Rev Cubana Pediatr. 2012;84(1):1-10. Available from http://scielo.sld.cu/scielo.php?script=sci_ arttext\&pid=S0034-75312012000100001\& $\operatorname{lng}=$ es.

7. Chung S, Onuzuruike A, Magge S. Cardiometabolic risk in obese children. Ann NY Acad Sci. 2018;1411(1):166-83. Available from https:// www.ncbi.nlm.nih.gov/pubmed/29377201

8. Jung M, Yoo E. Hypertriglyceridemia in obese children and adolescents.J Obes Metab Syndr. 2018;27(3):143-9. Available from https://www. ncbi.nlm.nih.gov/pubmed/31089556
9. Chiarpenello J. et al. Complicaciones endocrinometabólicas de la obesidad en niños y adolescentes. Rev. Med. Rosario. 2013;79:112-7. Available from http://www.circulomedicorosario.org/Upload/Directos/Revista/7ae25bChiarpenello\%20Complic\%20Endocr\%20 Obes\%20Ped\%20RMR\%202013.pdf

10. Martos G. et al. Las alteraciones metabólicas asociadas a la obesidad ya están presentes en los primeros ańos de vida: estudio colaborativo español. Nutr Hosp. 2014;30(4):787-93.

11. Almanzar R. 2016. Factores pronósticos del sindrome metabólico en obesidad de pacientes de 1 a 15 años. Tesis de Postgrado Endocrinología Pediátrica. Hospital Infantil Doctor Robert Reid Cabral. Santo Domingo, República Dominicana.

12. Francesco Chiarelli, Angelika Mohn. Early diagnosis of metabolic syndrome in children. The Lancet Child \& Adolescent Health. 2017; (1):286-8.

13. Blancas-Flores G, Almanza-Pérez JC, LópezRoa RI, Alarcón-Aguilar FJ, García-Macedo R, Cruz M. La obesidad como un proceso inflamatorio. Bol. Med. Hosp. Infant. Mex. 2010;67(2):88-97. Available from http://www. scielo.org.mx/scielo.php?script=sci_arttext \& pid=S1665-11462010000200002\&lng=es.

14. Moreno L, Alonzo M. Obesidad. Protocolos de la Asociación Española de Pediatría. 2da ed; 2010. Available from https://www.aeped.es/ documentos/protocolos-gastroenterologia-hepatologia-y-nutricion-en-revision

15. Organización Mundial de la Salud. Informe de Obesidad. 2017. Available from http://www. who.int/end-childhood-obesity/facts/es 